\title{
A Systematic Mapping Study of Value-based Software Engineering
}

\author{
Norsaremah Salleh \\ Computer Science Department \\ International Islamic University \\ Malaysia \\ norsaremah@iium.edu.my
}

\author{
Fabiana Mendes \\ Faculty of Information Technology \\ \& Electrical Engineering, \\ University of Oulu, Finland \\ fabiana.mendes@oulu
}

\author{
Emilia Mendes \\ Faculty of Computing, \\ Blekinge Institute of Technology, \\ Karlskrona, Sweden. \\ emilia.mendes@bth.se
}

\begin{abstract}
Integrating value-oriented perspectives into the principles and practices of software engineering is critical to ensure that software development and management activities address all key stakeholders' views and also balance short-and-long-term goals. This is put forward in the discipline of Value-Based Software Engineering (VBSE). In this paper, a mapping study of VBSE is detailed. We classify evidence on VBSE principles and practices, research methods, and the research types. This mapping study includes 134 studies located from online searches, and backward snowballing of references. Our results show that VB Requirements Engineering (22\%) and VB Planning and Control (19\%) were the two principles and practices mostly investigated in the VBSE literature, whereas VB Risk Management, VB People Management and Value Creation (3\% respectively) were the three less researched. In terms of the research method, the most commonly employed method is case-study research. In terms of research types, most of the studies $(28 \%)$ proposed solution technique(s) without empirical validation.
\end{abstract}

Keywords - Value-based software engineering, systematic mappping, VBSE principles and practices.

\section{INTRODUCTION}

Value-based Software Engineering (VBSE) integrates value thinking into Software Engineering (SE) principles and practices [1], and in this way aims to improve projects' outcomes [1],[2]. Value here refers to "relative worth, utility, or importance" [2], rather than "monetary worth of something: market price" [3]. Some of its principles are:

- Requirements/use cases/objects/defects/etc. should cease to be treated as equally important;

- Decisions should not only be based on project costs and schedule, but also on stakeholder or business value

Boehm (2003) also argued that a value perspective should be integrated into the full range of existing and emerging SE principles and practices, such as value-based requirements engineering, architecting, design and development, verification and validation, planning and control, risk management, quality management, and people management [1].

Since Boehm's seminal paper, other VBSE publications followed, investigating value-based approaches and techniques in SE (e.g. [4], [5], [6]); and also incorporating a value dimension in software development activities, such as requirements engineering (e.g. [7],[8]) and software quality assurance (e.g. [9]), to name a few.

The goal and main contribution of this paper is to present a mapping study (MS) aimed to provide an overview of VBSE, based on a representative sample of studies published from 2003 to 2017. Our MS structures the VBSE body of knowledge through a systematic classification of evidence based on the VBSE principles documented in [1]. Our MS has nine research questions (RQs); however, herein we provide the results for RQs 1, 2 and 3 (Section III). There are additional 6 RQs that were not included due to lack of space.

Therefore, this MS's key contributions are to: i) identify \& summarize trends in the VBSE research and possible opportunities for future research; ii) identify trends in VBSE research, and; iii) possible issues and advantages for the use of research methods and the research types.

The remainder of this paper is organized as follows: Section II presents related work, followed by the description of our MS process in Section III. Section IV presents our results followed by a discussion and threats to validity in Section V. Finally, Section VI concludes our work.

\section{RELATED WORK}

Although there has been an edited book on VBSE [2], this section focuses on discussing similarities and differences between our work and another two mapping studies and a systematic literature review, for being the closest to our work. Jan and Ibrar (2010) detailed a MS (MS1) where 364 studies, published between 1990 and 2010, were added in [10]. The differences between MS1 and ours are:

- Unlike ours, MS1 also included studies that did not comply with the VBSE definition in [1].

- We included studies published between 2003 and up until October 2017, while MS1 included studies published between 1999 and 2010.

- MS1 used a search string containing keywords from business, economics, management, finance, marketing and software engineering; ours used as 
keywords terms already employed in other VBSE studies we were aware of.

The second MS related to ours (MS2), by Khurum et al. [11], had a narrower focus than ours. They aimed to identify value propositions used when making decisions relating to software \& software-intensive products. Like MS1, they also included primary studies outside VBSE, published in fields such as economics and marketing.

The third and last study related to ours is a literature review (not carried out systematically) by Khan and Khan [12]. It focuses upon the impact that the adoption of a "value-based" approach to SE had upon software reusability and quality. Their analysis, limited to only ten (10) studies, presents different value-based pricing criteria, selection of automated tools, Component off the shelf (COTS), and contrasts between stakeholders' value propositions. They reference Barry Boehm's work; however they see value as solely financial, monetary.

\section{SYSTEMATIC MAPPING PROCESS}

This MS used the MS guidelines by Petersen et al. (2008) [13], and Petersen et al. (2015) [14]. Its activities, shown in Fig. 1, consist of three phases: planning, executing and reporting. The diagram also identifies in which activity each author participated - 1 for first author, 2 to second author, and 3 for third author.

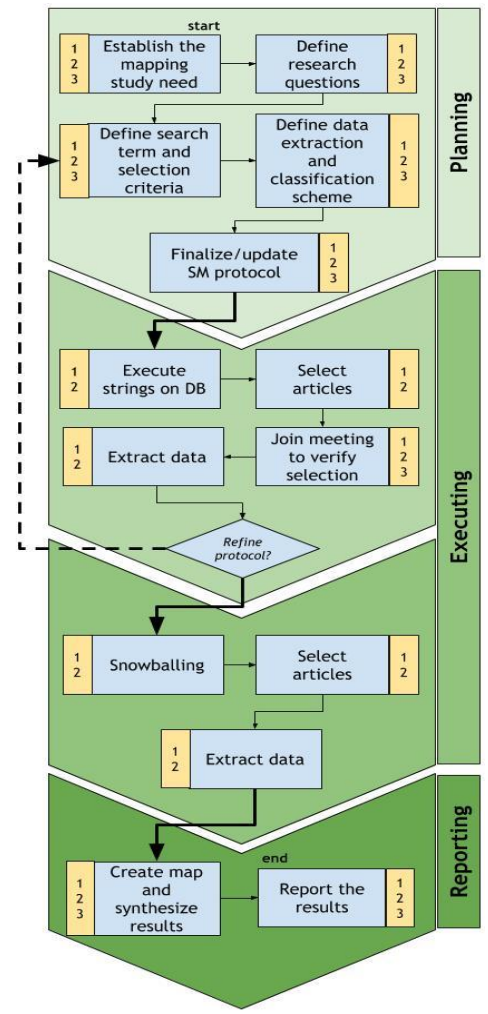

Fig. 1: Systematic Mapping Process

The planning phase relates to making decisions such as identifying the mapping study's goals, scope, research question, search strategy, selection criteria, extraction, and classification process. The next phase - Executing, includes all the processes that relate to the mapping study's execution and data recording. The last phase Reporting, represents the reporting and the results evaluation. Note that whenever there were any changes required either in the selection or extraction of studies, the mapping study protocol was updated accordingly.

\section{A. Research Questions}

This MS is guided by the following research questions:

- RQ1: What do we know about the SE principles \& practices investigated so far in VBSE?

- RQ2: What are the research methods used in VBSE studies and how many studies looked at each method (e.g. case study, experiments, survey etc.)?

- RQ3: What are the research types that these studies apply (e.g. validation /evaluation/ solution proposal etc.) and how many studies looked at each research type?

\section{B. Search Strategy}

We used the following search string:

(("value-based" AND "software engineering") OR ("value-based software engineering") OR ("value based" $A N D$ "software engineering") $O R$ ("Value based software engineering") OR VBSE) OR ((value OR "value based" OR "valuation" $O R$ "value creation") $A N D$ ("economics based" OR "decision making" OR economics OR "software project") AND ("software engineering" OR software OR "software development"))

Petersen et al. (2015) present three different search strategies for MSs: automatic search on electronic databases, manual search and snowballing. In this MS, we have conducted an automatic search on electronic databases, complemented with snowballing [15]. For the electronic searches, we selected articles published until up to $18^{\text {th }}$ October 2017 (i.e. the date we executed the search on online databases).

We included online databases that indexed each of the six VBSE papers known prior to conducting this MS. In addition, there were also previous systematic literature reviews (SLRs) and MSs that provide recommendations on the most adequate online databases to use (e.g. [16],[17]). Based upon both, we decided to use the following databases: IEEEXplore, ACM digital library, Scopus, ScienceDirect, ISI Web of Science, and SpringerLink.

Note that although there are a few other potential databases such as EI compendex, Wiley Interscience (Wiley Online), Inspec and Kluwer as identified in [17], these databases were excluded due to the high degree of overlap among the databases, as reported by [16]. 


\section{Study Selection}

Petersen et al. (2015) [14] suggest that both the inclusion and exclusion criteria should consider: "(a) the relevance of the topic of the article, (b) the venue of publication, (c) the time period considered, (d) requirements on evaluation, and (e) restrictions with respect to language". However, they add that a MS should not consider item (d) so to avoid excluding studies that have not yet reached the maturity for evaluation. This guideline, and examples provided in [14] motivated the selection criteria presented in Table I.

During the study selection, the full text was referred to in most cases. Further, a number of papers were rejected due to using value solely as synonym to shortterm aspects such as costs, schedule, and effort.

\section{TABLE I. INCLUSION \& EXCLUSION CRITERIA}

\begin{tabular}{|l|}
\hline \multicolumn{1}{|c|}{ Inclusion Criteria } \\
\hline IC 01 - The study is directly related to VBSE \\
\hline \multicolumn{1}{|c|}{ Exclusion Criteria } \\
\hline EC 01 - The study lies outside the SE domain \\
EC 02 - The study is grey literature (e.g. thesis) \\
EC 03 - The study is not written in English \\
EC 04 - The study's full text is not available \\
EC 05 - The study is within the SE domain but does not relate to \\
VBSE
\end{tabular}

\section{Classification Scheme}

To create a map of VBSE publications, we applied two approaches, as suggested by [14]: topic-independent (general) classification and topic-dependent classification. With regard to the former, we arranged studies according to research topic, publication venue, research method, research type, study context, contribution facet, citation and publication count. In relation to the latter, we adopted the research agenda proposed by [1].

We used the classification of research methods as reported in [19], [20] and [21], as follows: Controlled experiment, case study, survey research, ethnography, action research, simulation, prototyping, mathematical analysis, mathematical proof properties, literature review, and mixed method.

Classification of research type followed the existing types of research approaches suggested by [19], as follows: evaluation, experience report, opinion paper, philosophical paper, solution proposal, and validation. Evaluation research refers to studies that performed evaluation on techniques that have been implemented in practice. Validation research refers to studies that investigated techniques that have not yet been applied in practice. Experience study reports how something was done in practice as a result of the author's personal experience. Opinion paper reports the author's opinion about something (e.g. how something should be done). Philosophical paper reports original conceptual framework or a new way of looking at things. Solution proposal reports a solution technique and argues about its relevance without a full-blown validation. Table II presents both types of classification used herein to structure the VBSE literature.

TABLE II. CLASSIFICATION SCHEMES

\begin{tabular}{|l|l|}
\hline General Classification & Topic-dependent Classification \\
\hline \multirow{3}{*}{ - Research method } & - Value-based requirements engineering \\
- Research types & - Value-based architecting \\
& - Value-based design and development \\
& - Value-based planning and control \\
& - Value-based risk management \\
& - Value-based quality management \\
& - Value-based people management \\
& - A theory of VBSE \\
\hline
\end{tabular}

IV. RESULTS

The search from online databases retrieved a total of 6308 studies. Table III shows the results of our automated search process. Out of 6308, we selected 117 studies that fulfilled the inclusion and exclusion criteria. We further conducted one iteration of backward snowballing where out of 3273 references from 117 studies, we selected another 17 studies. This led to a total of 134 studies $^{1}$.

TABLE III. SEARCH RESUlt From ONLINE DATABASES

\begin{tabular}{|l|c|c|c|}
\hline Database & Retrieved $\left(^{*}\right)$ & Excluded & Included \\
\hline ACM & 200 & 190 & 10 \\
\hline IEEE & 1258 & 1230 & 28 \\
\hline ISI WoS & 1005 & 979 & 26 \\
\hline Science Direct & 89 & 89 & 0 \\
\hline Scopus & 1567 & 1517 & 50 \\
\hline SpringerLink & 2189 & 2186 & 3 \\
\hline TOTAL & 6308 & 6191 & 117 \\
\hline
\end{tabular}

Fig. 2 shows the number of studies that were published each year, since 2003 .

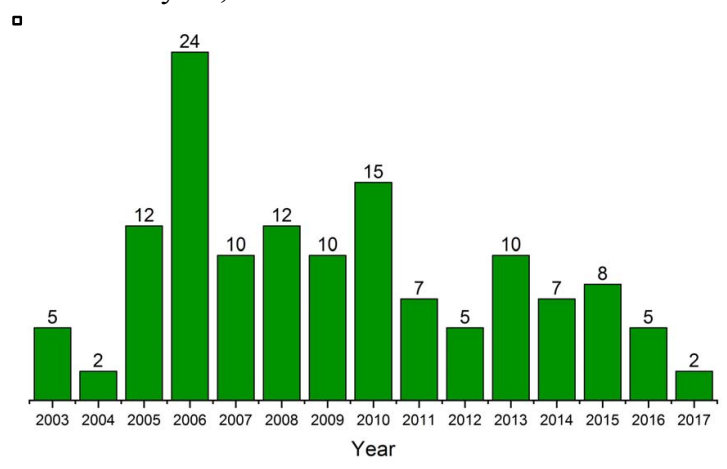

Fig. 2: Number of publications per year

The pattern shows that during the first two years (2003 and 2004) there were only jointly seven studies published; publication numbers peaked in 2006 with 24 studies (12 of these were book chapters in the VBSE book by [2]. Overall, we have seven years with at least 10 publications per year, followed by another five years with at least 5

\footnotetext{
${ }^{1}$ The list of 134 studies is available here: https://sites.google.com/view/vbsems/home
} 
publications per year. Of these 134 studies, 32 (24\%) were published in journals, $84(62 \%)$ in conference proceedings and the remaining $18(14 \%)$ as book chapters.

\section{A. Answering Research Question 1}

What do we know about the SE principles \& practices investigated so far in VBSE?

To answer the first RQ we used as basis the classification suggested in Boehm's VBSE Agenda for existing and emerging SE principles and practices [1] (requirements engineering, architecting, design \& development, verification \& validation, planning \& control, risk-management, quality management, people management, and Theory of VBSE), plus an additional four practices not included in the original agenda (valuebased decision-making, software process, value creation and a fourth category called 'Other' (i.e. studies looking at general aspects of VBSE)). These latter practices were added in order to be able to classify all the selected studies. Table IV provides details relating to which studies belong to a given category.

TABLE IV. VBSE AGENDA

\begin{tabular}{|l|l|c|}
\hline VBSE Agenda & \multicolumn{1}{|c|}{ Paper ID } & Total \\
\hline $\begin{array}{l}\text { VB } \\
\text { Enguirements }\end{array}$ & $\begin{array}{l}\text { S7, S8, S13, S14, S15, S18, S19, S21, } \\
\text { S65, S75, S55, S56, S60, S61, S62, } \\
\text { S103, S104, S107, S109, S116, S119, } \\
\text { S126, S130 }\end{array}$ & 30 \\
\hline $\begin{array}{l}\text { VB Planning and } \\
\text { Control }\end{array}$ & $\begin{array}{l}\text { S20, S23, S24, S30, S32, S34, S36, } \\
\text { S39, S40, S43, S51, S68, S70, S75, } \\
\text { S81, S92, S95, S96, S105, S108, } \\
\text { S111, S118, S123, S131, S134 }\end{array}$ & 25 \\
\hline $\begin{array}{l}\text { VB Quality } \\
\text { Management }\end{array}$ & $\begin{array}{l}\text { S10, S11, S16, S17, S22, S27, S31, } \\
\text { S11, S53, S57, S74, S91, S94, S113, }\end{array}$ & 16 \\
\hline $\begin{array}{l}\text { VB Design and } \\
\text { Development }\end{array}$ & $\begin{array}{l}\text { S25, S37, S52, S63, S64, S66, S77, } \\
\text { S78, S106, S129, S132 }\end{array}$ & 11 \\
\hline $\begin{array}{l}\text { VB Verification } \\
\text { \& Validation }\end{array}$ & $\begin{array}{l}\text { S6, S46, S59, S71, S73, S83, S93, } \\
\text { S98, S100 }\end{array}$ & 9 \\
\hline $\begin{array}{l}\text { VB Decision } \\
\text { Making }\end{array}$ & S54, S84, S88, S90, S112, S117, S124 & 7 \\
\hline VB Architecting & S12, S58, S80, S85, S89, S122, S133 & 7 \\
\hline $\begin{array}{l}\text { VB Software } \\
\text { Process }\end{array}$ & S5, S33, S42, S44, S87, S120 & 6 \\
\hline Theory of VBSE & S1, S4, S48, S49, S50, S69, S76 & 7 \\
\hline $\begin{array}{l}\text { VB Risk } \\
\text { Management }\end{array}$ & S29, S47, S102, S121 & 4 \\
\hline $\begin{array}{l}\text { VB People } \\
\text { Management }\end{array}$ & S2, S38, S45, S128 & 4 \\
\hline Value Creation & S9, S110, S115, S127 & 4 \\
\hline Other & S3, S26, S67, S82 & 4 \\
\hline
\end{tabular}

Results show that VB Requirements Engineering (RE) has been VBSE's mostly investigated principle \& practice, contributing with 30 studies $(22.4 \%)$. Out of these 30 studies, 11 studies proposed a value-based method, or approaches to prioritize requirements ([S7],[S8],[S13],[S14],[S19],[S35],[S60],[S65],[S72],[S1 16],[S119]); four (4) studies proposed a model and method to support requirements elicitation activities ([S18],[S56],[S61],[S62]); three (3) studies ([S28],[S55],[S97]) conducted surveys to identify stakeholders' requirements prioritization criteria; three (3) studies used an RE process to focus upon software product value ([S79],[S101],[S104]); two (2) studies ([S99],[S109]) proposed requirements negotiation's method and model, two (2) studies ([S107],[S126]) presented practitioners-based requirements selection decision-making criteria; the remaining five (5) presented various approaches to assist requirements activities ( $\mathrm{S} 15$, S21, S86, S103, S130).

The second most investigated VBSE principle \& practice was VB Planning \& Control (25 studies). Nine studies (9 studies) proposed ways to support organizations plan software projects ([S20], [S68], [S70], [S95], [S40], [S23], [S105],[S118],[S134]); three studies ([S32],[S96],[S123]) proposed value-based methods to support release planning; two studies ([S24],[S111]) focused on stakeholder value; the remaining eleven (11) studies focused on assisting project planning in general (S39, S43, S36, S81, S92, S108, S30, S34, S51, S75, S131).

VB Quality Management research mainly focused on software processes' quality aspects ([S11], [S17], [S27], [S31]). S11 proposed a conversion mechanism from a traditional to a value-based (VB) software development process, while S17 analyzed quantitatively VB software processes using Decision-based Stochastic Object PetriNets. S31 proposed a software process framework using the $4+1$ theory [18], while S27 surveyed the relationship between software project selection process and the business strategy. Studies [S10],[S91] investigated quality alignment between key stakeholders. Studies [S16], [S113], [S125], [S114] reported software quality assurance value aspects. In S53, the Value-based software quality achievement (VBSQA) process was tailored to different business cases; in [S57] they demonstrated the use of VBSQA in an Enterprise Resource Planning software project in China. The remaining four (4) studies focused on software quality investment [S22], software dependability analysis [S41], quality processes assessment using OCD-COQUALMO [S74], and multiattribute decision support techniques [S94].

VB Design and Development includes techniques and approaches to ensure value-considerations are integrated into a software's design and development [1]. Three (3) out of 11 studies proposed a mechanism and prototype tool for stakeholders' value realization and to add a valuechain in software component markets [S78], [S63], [S129]. The remaining eight (8) studies proposed: i) a design technique to estimate a design strategy's value [S66], ii) a context-based approach for decision support systems [S52], iii) a method to include customers' value when designing embedded systems [S64], iv) guidelines on how to tame inconsistencies during software development [S25], v) a method to capture antagonistic stakeholders value propositions [S37], vi) a value decomposition model catering to customers' and the organization's values [S77], vii) a method to guide a 
software design improvement [S106], and viii) an approach to reasoning about software designs' value [S132].

VB Verification and Validation (V\&V) was the target of nine (9) studies. Two (2) of these focused on prioritization strategies to improve software testing cost-effectiveness [S98], [S83]. A value-based software testing method was proposed in S93. In [S100], authors applied OCD COQUALMO model with machine learning and strategic optimization to enhance the $\mathrm{V} \& \mathrm{~V}$ process. Other suggested approaches include: software system testing's coverage measurement tool [S6] and value-based management [S46], and software evolutionary testing framework using genetic algorithms [S59]. Two (2) studies [S71], [S73] compared the performance of valuebased review (VBR) with the traditional value-neutral checklist based reading (CBR) approach

Seven (7) studies were on VB Decision-Making. Two (2) studies [S84], [S88] looked at feature usage measures and customers' product features' perceived value to support decision-making. S90 proposed a software value map for making decisions about product management and development. In more recent studies, [S54], [S112] introduced a VALUE framework to estimate the value associated with stakeholders' decisions related to software product/project management and development. They also developed a Value tool to support a decision-making process [S117]. In [S124], the authors performed classification of value aspects in project management context.

Seven (7) studies relate to VB Architecting: Three (3) [S58], [S80], [S85] focused on a value-based approach for documenting design-decisions rationale. The other studies proposed: a customer-centric value for assessing system architecture investment [S89], a lightweight value-based architecture evaluation (LiVASAE) [S12], a value discovery method in the context of Big Data design [S122], and a method to evaluate diversification of software architecture [S133].

Six (6) studies focused at the VB Software process: Two (2) explored VB factors' impact upon software development process [S5], [S33]. S87 conducted a survey to identify value factors used in software development; results informed a framework for software process tailoring [S120]. S44 introduced a software process model and S42 put forward a value-based set of processes for Components-Off-The-Shelf (COTS)-based applications. Three of the seven (7) studies classified under Theory of VBSE described the 4+1 theory [S48], [S69], [S76]. One study [S4] analyzed several software implementation projects and proposed a VBSE theory's extension. The remaining three (3) studies [S1], [S49], [S50] present the VBSE agenda including the VBSE elements.

The four VB Risk Management included: valuation of software initiatives under uncertainty [S47], a value-based process to manage requirements-related risks [S29], a component-based system development's architectural mismatches' risk assessment model [S102], and a software project's uncertainty measurement method [S121].

The four (4) studies in VB People Management described four different aspects: value-based knowledge management to support learning in software companies [S2], value-based approach for managing architectural knowledge [S38], collaborative process to facilitate stakeholders' involvement [S45], and stakeholder value to understand conflicts in software development [S128].

Value Creation included four (4) studies: i) challenges in customer value creation in global SE [S9], ii) establishing value of software through a new definition of value [S110], iii) value creation in agile project [S115], and iv) measuring economic value creation in agile methods [S127].

The remaining four (4) papers in the "Other" category are general VBSE papers: i) a framework to identify and to estimate new innovation idea's value [S3], ii) the applicability of Lean Six-sigma principles for a VBSE process [S82], iii) applications of machine learning methods in VBSE [S26], and iv) an educational game as a pedagogical aid in teaching VBSE to students [S67].

A bubble plot of VBSE principles \& practices vs year (see Fig. 3) shows the overall trends of publications. The bubbles' size represents the amount of papers published, and the numbers near bubbles are the number of papers. Based on publication number, results suggest constant interest in VB Requirements Engineering research, and an emerging interest in VB decision-making research, and VB Software process. The VB Planning and Control has been actively researched until 2010 and the trend is decreasing from 2011 onwards. Similarly, there seems to be a decrease in research related to the VBSE theory, in which the last publication appeared in 2011. There is no available publication in VB Risk Management and Value Creation from 2011 onwards, whereas for VB People Management, the last publication appeared in 2010. The trend also indicates no publication on $\mathrm{VB} V \& \mathrm{~V}$ from 2014 onwards. 


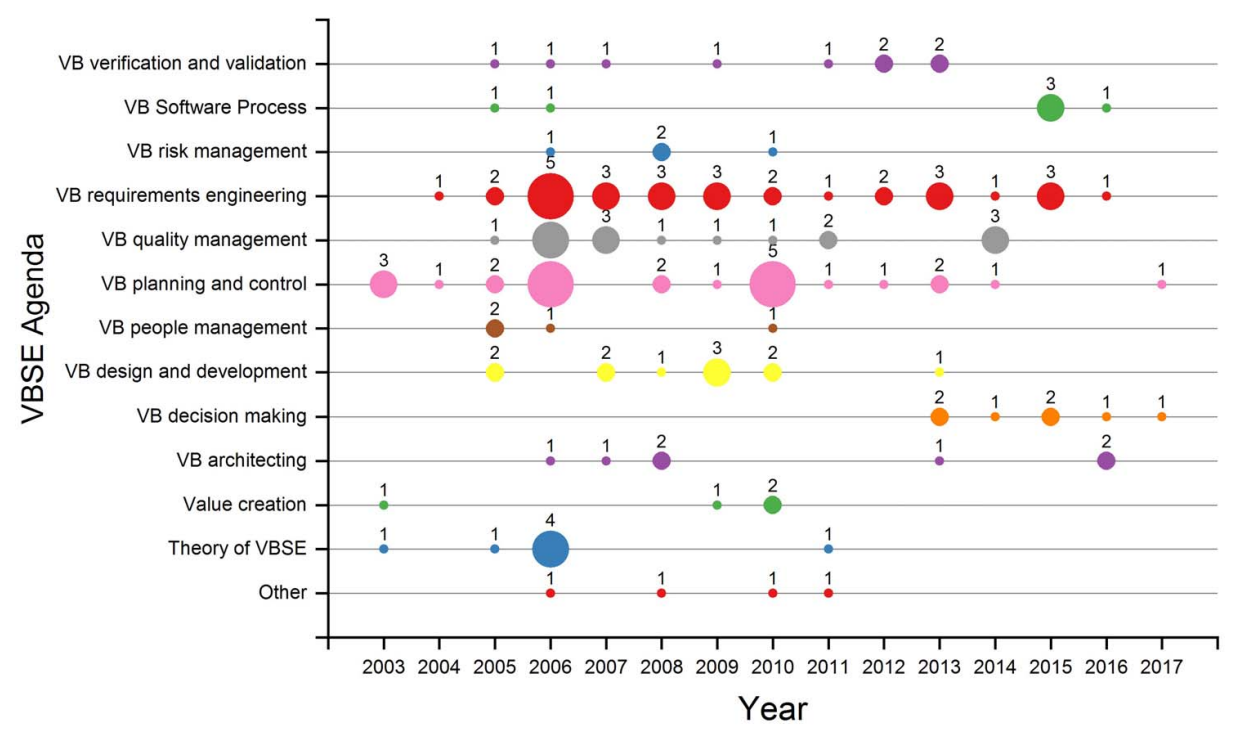

Fig. 3: VBSE Principles \& Practices papers per year

\section{B. Answering Research Question 2}

What are the research methods used in VBSE studies and how many studies looked at each method (e.g. case study, experiments, survey etc.)?

To answer this question we used the research methods' classifications in [19], [20], and [21] (see Table $\mathrm{V})$.

TABLE V. BREAKDOWN OF STUDIES BY RESEARCH METHODS

\begin{tabular}{|l|l|}
\hline Method & \multicolumn{1}{|c|}{ Studies } \\
\hline $\begin{array}{l}\text { Case Study } \\
(51)\end{array}$ & S8, S9, S10, S14, S18, S19, S21, S23, S24, \\
S30, S31, S39, S40, S41, S43, S50, S53,S56, \\
S60, S61, S68, S70, S72, S76, S79, S83, S84, \\
S86, S88, S89, S91, S93, S98, S103, S105, \\
S115, S117, S119, S121, S122, S123, S131, \\
S29, S95, S109, S102, S108,S130, S17, S64, \\
S133
\end{tabular}

Our analysis showed that 51 studies (38\%) were conducted using a case-study methodology. Forty-two
$(31.3 \%)$ carried out their case studies within an organizational context (e.g. defense agency [S56], software organization [S83], startup company [S84], [S88] and large company such as Ericsson [S91]); eighteen studies (13.4\%) proposed a solution without empirical validation or evaluation (e.g. S6, S46, S62). Eleven studies $(8.2 \%)$ used a combination of research methods (mixed-method), and were mostly performed in an organizational context, with only two (1\%) in industry [S54], [S104]. Surveys and controlled experiments were reported in eleven (8.2\%) and ten (7.5\%) studies respectively, mostly conducted in an organizational or academic setting. S87 is the only survey executed in a government context. Finally, there are a small number of studies that performed simulations (S52, S111), discourse analysis (S77), literature reviews (S33, S34, S124) and action research $(\mathrm{S} 2, \mathrm{~S} 45)$.

\section{Answering Research Question 3}

What are the research types that these studies apply (e.g. validation /evaluation/solution proposal etc.) and how many studies looked at each research type?

To answer this question, we classified studies according to the types of research approaches by [19] (see Table VI). A total of 38 studies (28\%) proposed solution technique(s) without any empirical validation or evaluation; next, another 34 studies (25.3\%) presented solution proposals together with a validation strategy. The remaining studies were categorized into evaluation (20 studies, 14.9\%), validation (16 studies, 11.9\%), solution proposal and evaluation (7 studies, 5.2\%), philosophical papers (8 studies, 6\%), experience report (6 studies, $4.5 \%$ ), literature review (4 studies, 3\%), and opinion (3 studies, $2.2 \%$ ).

The bubble plot in Fig. 4 shows the dimensions for assessing the relationship between the VBSE agenda and 
research type. As can be seen, a clear majority of valuebased studies in Requirements Engineering, Planning \& Control, and Design \& Development presented either a solution proposal or solution proposal together with a validation. Analysis showed that there is a lack of validation research in VB software process, people management, design \& development, value creation, and VBSE theory. There is also no evidence available for evaluation type of studies in VB V \&V, risk management, design \& development, architecting, and VBSE theory. This may suggest that a lack of empirical evaluation actually takes place in a real-world industrial context for research in these areas. There are also very little studies reporting experiences in implementing VBSE in industry.

TABLE VI. BREAKDOWN OF STUDIES BY RESEARCH TYPES

\begin{tabular}{|l|l|}
\hline Research Type & Studies \\
\hline $\begin{array}{l}\text { Solution Proposal } \\
(38)\end{array}$ & S2, S3, S5, S8, S13, S20, S22, S25, S32, S36, \\
& S37, S42, S44, S45, S46,S51, S52, S54, S59, S62, \\
S63, S64, S66, S67, S74, S78, S81, S92, S101, \\
S106, S110, S111, S118, S120, S127, S129, \\
S132, S134
\end{tabular}

-

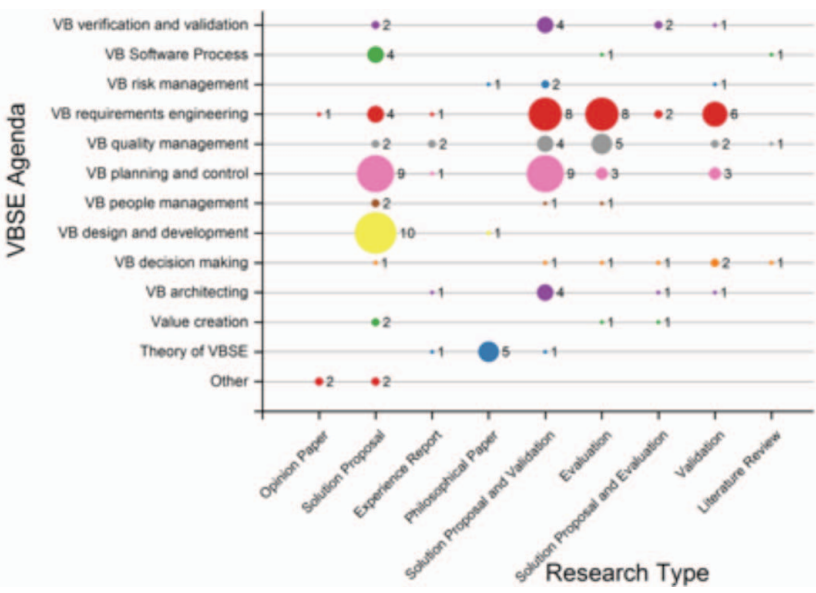

Fig. 4: VBSE Agenda by Research Type

\section{DISCUSSION \& THREATS TO VALIDITY}

One of the fundamental results is the identification of VB Requirements Engineering and VB Planning \& Control as the two significant areas constantly being researched since the period of 14 years. Thirty (30) studies focused on various topics related to integrating value perspectives in requirements engineering, particularly looking at methods, processes and techniques to elicit and reconcile stakeholder's value proposition in prioritizing requirements (e.g. S7, S13, S19). This finding concurs with the results from a previous study reported by [10]. Further, we observed that most studies $(60 \%)$ performed proposal solutions, validation, or both, hence very little empirical evaluation studies available in this principle and practice. Similar findings applied to studies related to VB Planning \& Control, where only three of them (12\%) performed empirical evaluation (S30, S34, and S108).

Overall, results indicate low maturity level of evidence due to lack of empirical evaluation in implementing the proposed solutions. More experimental research is needed to evaluate the suggested approaches and their effectiveness in a real industrial setting. This applies particularly to VB V\&V, VB Risk Management, VB Design \& Development and VB Architecting.

Our results show that the areas less researched are VB Risk Management, VB People Management, and Value Creation. VB Risk Management concerns on principles and practices for risk identification, analysis, prioritization, and mitigation [1]. It is indeed vital for an organization to have a strategy or approach to manage risks by integrating stakeholders' value considerations [22]. One of the suggestions to address risk is to apply the principles from the Incremental Commitment Spiral Model (ICSM) [23]. Further, we hardly found studies that assessed risk in software projects; only one (1) study (S102) utilized the Incremental Commitment Model (ICM) to improve project monitoring based on balancing opportunities and risks.

The lack of research in these three areas (Risk Management, People Management and Value Creation) indicates an opportunity to conduct new research undertakings particularly in mitigating project risks and strengthening or improving the stakeholders' team building and/or decision-making. One particular aspect important to be investigated, for example, is to explore the role of stakeholders' personality in performing valuebased decision-making. This could help improve the quality of decision-making process of stakeholders' team.

One of the major threats for this mapping study is the possibility of missing relevant evidence. This was mitigated via two search phases, which included electronic online databases search and backward snowballing. During the online database search, we applied a more generic search terms consist of the combination of VBSE keywords including relevant synonyms.

Our strategy to complement the search was therefore to conduct the snowballing for all included studies. Based on the manual filtering of 3273 references from 117 primary studies, the snowballing helped discover another 17 studies. We believe we have included herein studies that 
represent a significant sample of the VBSE research population.

\section{CONCLUSIONS}

This paper presents the results for three RQs from a mapping study in VBSE that included 134 studies. It aims to provide a preliminary overview of VBSE research to support SE researchers and practitioners through a collection and systematic classification of VBSE studies.

Results showed that VB Requirements Engineering (22\%) and VB Planning and Control (19\%) were the two principles and practices mostly researched in VBSE literature, whereas VB Risk Management, VB People Management and Value Creation were the less researched (3\% respectively). Studies in VB Requirements Engineering mostly focused on proposing new methods, processes and techniques for prioritizing requirements and mechanisms to elicit and reconcile stakeholder's value propositions.

In terms of the research method, $38 \%$ of the studies used case-study methodology, hence it appears to be the most common method employed. Other methods used were surveys, experiments, action research, prototyping, literature review, quantitative analysis, simulation, and mixed method. Approximately $26 \%$ of the studies did not declare or did not report empirical findings.

There are a small number of evaluation studies available in VBSE literature with only $18 \%$ of the studies perform evaluation of the proposed solution within the industry or organizational context. A large number of studies (approx. 54\%) either presented only the solution proposal or solution together with the validation.

As part of our future work we seek to investigate how the value-based decision-making process could be influenced by the stakeholders' personality. This could potentially help address the lack of research in VB People Management in the effort to improving stakeholders' decision-making and strengthening the team building

\section{ACKNOWLEDGMENTS}

This research is funded by Tekes under the FiDiPro VALUE project and the Ministry of Higher Education Malaysia under FRGS/1/2018/ICT01/UIAM/02/2 grant.

\section{REFERENCES}

[1] B. Boehm, "Value-based software engineering," ACM SIGSOFT Software Engineering Notes, Vol. 28, No. 2, pp. 1-12, March 2003

[2] S. Biffl, A. Aurum, B. Boehm, H. Erdogmus, and P. Grünbacher, editors. Value-based software engineering. Berlin, Germany: Springer Science \& Business Media; 2006 Feb 23. Doi: 10.1007/33-540-29263-2

[3] Webster. 2016. In Merriam-Webster.com. Retrieved Dec. 23, 2016, from https://www.merriam-webster.com/dictionary/value.

[4] L. Huang, "A value-based process for achieving software dependability," International Software Process Workshop, pp. 108-121, May 2005.

[5] X. Zhang, G. Auriol, and C. Baron, "Understanding customer expectation for system development," 5th International Conf. on Software Engineering Advances (ICSEA), 2010.

[6] E. Mendes, B. Turhan, P. Rodriguez, and V. Freitas, "Estimating the value of decisions relating to managing and developing software intensive products and projects," Proceedings of the 11th International Conf. on Predictive Models and Data Analytics in Software Engineering (PROMISE '15), 2015.

[7] N. Kukreja, B. Boehm, S.S. Payyavula, S. Padmanabhuni, "Selecting an Appropriate Framework for Value-Based Requirements Prioritization A Case Study," 21st IEEE International Conf. on Requirements Engineering (RE), 2012, doi:10.1109/RE.2012.6345819.

[8] F. Sher, D.N.A. Jawawi, R. Mohamad and M.I. Babar, "Multiaspects based requirements prioritization technique for value-based software developments," International Conf. on Emerging Technologies (ICET), pp. 1 - 6, 2014.

[9] D. Port, J. Wilf, M. Diep, C. Seaman, and M. Feather, "Developing a Value-Based Methodology for Satisfying NASA Software Assurance Requirements," Proc. of the 49th Hawaii International Conf. on System Sciences (HICSS), pp. 5642 - 5651, 2016.

[10] N. Jan and M. Ibrar, "Systematic Mapping of Value-based Software Engineering - A Systematic Review of Value-based Requirements Engineering," Masters thesis Software Engineering, thesis number: MSE-2010:40, Blekinge Institute of Technology, Sweden., 2010.

[11] M. Khurum, T. Gorschek, and M. Wilson, "The software value map - an exhaustive collection of value aspects for the development of software intensive products," Journal of Software: Evolution and Process, vol. 25, no. 7, pp. 711 - 741, Jul 2013.

[12] M.Z. Khan and M.N.A. Khan, "A review of value-based software engineering and its impacts," International Journal of Advanced Science and Technology, vol. 75 (2015), pp. 33-42, 2015.

[13] K. Petersen, R. Feldt, S. Mujtaba, and M. Mattsson, "Systematic mapping studies in software engineering," 12th International Conf. on evaluation and assessment in software (EASE'12), vol. 17, no. 1, June 2008.

[14] K. Petersen, S. Vakkalanka, and L. Kuzniarz, "Guidelines for conducting systematic mapping studies in software engineering: An update," Information and Software Technology, vol. 64, pp. 18, Aug. 2015.

[15] S. Jalali and C. Wohlin, "Systematic Literature Studies: Databases Searches vs Backward Snowballing," International Symposium on Empirical Software Engineering and Measurement - ESEM 2012, pp. $29-38,2012$

[16] D. Maplesden, E. Tempero, J. Hosking, and J.G. Grundy, "Performance analysis for object-oriented software: A systematic mapping," IEEE Trans. on Software Engineering, vol. 41, no. 7, 2015.

[17] H. Zhang, M.A Babar, and P. Tell, "Identifying relevant studies in software engineering," Information and Software Technology, vol. 53 , no. 6, pp. 625-637, Jun. 2011.

[18] B. Boehm and A. Jain, "An initial theory of value-based software engineering," Value-Based Software Engineering, S. Biffl, A. Aurum, B. Boehm, H. Erdogmus, P. Grünbacher, eds., Springer Berlin Heidelberg, pp. 15-37, 2006.

[19] R. Wieringa, N. Maiden, N. Mead, and C. Rolland, "Requirements engineering paper classification and evaluation criteria: a proposal and a discussion," Requirements Engineering, vol. 11, no. 1, pp. $102-107,2006$.

[20] S. Easterbrook, J. Singer, M.-A. Storey, D. Damian, Selecting empirical methods for software engineering research, in: Guide to Advanced Empirical Software Engineering, Springer, pp. 285-311, 2008.

[21] C. Wohlin, P. Runeson, M. Höst, M.C. Ohlsson, B. Regnell, and A. Wesslén, Experimentation in software engineering, Springer Science \& Business Media; June 2012.

[22] J. Samad, N. Ikram, and M. Usman, "VRRM: A value-based requirements' risk management process," IASTED International Conf. on Software Engineering, Austria, pp. 184 - 191, 2008.

[23] B. Boehm, J.A. Lane, S. Koolmanojwong, R. Turner, The Incremental Commitment Spiral Model: Principles and Practices for Successful Systems and Software, Pearson Education, Inc., 2014. 